\title{
Complete Analytical Solutions of the Mie-Type Potentials in $N$-Dimensions
}

\author{
D. AgBoolA* \\ Department of Mathematics, School of Mathematics and Physics, The University of Queensland \\ Brisbane QLD 4072, Australia
}

(Received July 3, 2010; in final form May 27, 2011)

\begin{abstract}
The exact solutions of the $N$-dimensional Schrödinger equation with the Mie-type potentials are obtained. The energy levels are worked out and the corresponding wave functions are obtained in terms of the Laguerre polynomial. Some energy levels of some diatomic molecules are given using the modified Kratzer-type potential. The expectation values $\left\langle r^{-1}\right\rangle$ and $\left\langle r^{-2}\right\rangle$ and the virial theorem are also obtained in $N$-dimensions using the Hellmann-Feynman theorem. The ladder operators are also constructed for the Mie-type potentials in $N$-dimensions and the matrix elements of some operators $r$ and $r \frac{\mathrm{d}}{\mathrm{d} r}$ are analytically obtained from the ladder operators. The general results reduce to the 3-dimensional case when $N=3$.
\end{abstract}

PACS: 03.65.- $\mathrm{w}$, 03.65.Fd, 03.65.Ge

\section{Introduction}

The exact bound-state solutions of the Schrödinger equation with physically significant potentials play a major role in quantum mechanics. Over the decades, exact solutions of the multidimensional Schrödinger equation have attracted much interest. Problems involving the N-dimensional Schrödinger equation have been severally solved by some researchers with the special transformation of the $N$-dimensional Schrödinger equation. For instance, Bateman et al. investigated the relationship between the hydrogen atom and a harmonic oscillator potential in arbitrary dimensions [1]. In a recent study [2], the exact solutions of the $D$-dimensional Schrödinger equation for an energy dependent potential have been presented by Hassanabadi and co-workers. Also, the $N$ -dimensional pseudoharmonic oscillator was discussed by Oyewumi et al. [3]. Recently, a $D$-dimensional study of both the Hulthén [4, 5] and Pöschl-Teller [6] potentials has been discussed by Agboola.

Recently, Chen and Dong [7] found a new ring-shaped potential and obtained the exact solution of the Schrödinger equation for the Coulomb potential plus this new ring-shaped potential which has possible applications to ring-shaped organic molecules like cyclic polyenes and benzene. Very recently, Cheng and Dai [8] proposed a new potential consisting of the modified Kratzer potential [9] plus the new proposed ring-shaped potential, and they presented the energy eigenvalues for this proposed exactly-solvable non-central potential in three-dimensional Schrödinger equation.

The path integral solution for one-dimensional special case Mie potential which is a perturbed Coulombic-

\footnotetext{
* e-mail: tomdavids2k6@yahoo.com
}

-type potential was obtained in [34]. Moreover, the Schrödinger equation for a system bound by a Mie-type potential was also solved by using the $1 / N$ expansion method [33]. In this paper, we give a complete normalized polynomial solution for the general $N$-dimensional Schrödinger equation for diatomic molecular systems interacting through Mie-type potential, which reduces to the standard three-dimensional case when the parameter $N$ is set equal to 3 . Moreover, we also present some quantum-mechanical properties such as some expectation values and the ladder operators of a system bound with the Mie-type potential in $N$-dimensions.

The work is arranged as follows. Section 2 gives the solution to the $N$-dimensional Schrödinger equation with the Mie-type potential and then the energy eigenvalues and the eigenfunctions are obtained. Also, in Sect. 3 and the next section some expectation values are dealt with the ladder operators for the Mie-type potential. Finally, we give the conclusions of the work in Sect. 5 .

\section{Schrödinger equation in $N$-dimensional spaces}

Using the $N$-dimensional polar coordinate with polar variable $r$ (hyper radius) and the angular momentum variable $\theta_{1}, \theta_{2}, \theta_{3}, \ldots, \theta_{N-2}, \phi$ (hyper angle), the Laplacian operator in the polar coordinate $r, \theta_{1}, \theta_{2}, \theta_{3}, \ldots, \theta_{N-2}, \phi$ of the $R^{N}$ is

$$
\nabla_{N}^{2}=r^{1-N} \frac{\partial}{\partial r}\left(r^{N-1} \frac{\partial}{\partial r}\right)+\frac{\Lambda_{N}^{2}(\Omega)}{r^{2}},
$$

where $\Lambda_{N}^{2}(\Omega)$ is a partial differential operator on the unit sphere $S^{N-1}$ (the Laplace-Betrami operator or the grand orbital operator) defined analogously to a three-dimensional angular momentum [18] as $\Lambda_{N}^{2}(\Omega)=-\Sigma_{i \geq j}^{N}\left(\Lambda_{i j}^{2}\right)$ where $\Lambda_{i j}^{2}=x_{i} \frac{\partial}{\partial x_{j}}-x_{j} \frac{\partial}{\partial x_{i}}$ for 
all Cartesian component $x_{i}$ of the $N$-dimensional vector $\left(x_{1}, x_{2}, \ldots, x_{N}\right)$.

The $N$-dimensional Schrödinger equation has the form $[1,2,19]$ :

$$
\begin{aligned}
& -\frac{\hbar^{2}}{2 \mu} \nabla_{N}^{2} \Psi_{n \ell m}(r, \Omega)+V(r) \Psi_{n \ell m}(r, \Omega) \\
& \quad=E \Psi_{n \ell m}(r, \Omega),
\end{aligned}
$$

where $\mu$ is the reduced mass and $\hbar$ is the Planck constant.

\subsection{Eigenvalues and eigenfunctions of the Mie-type potential}

The Mie-type potential, which is a diatomic potential, has been studied using methods such as the NikiforovUvarov Method [10], polynomial method [11-14], the ansatz wave function method $[15-17,20]$ and the $1 / N$ expansion method [33]. Generally, one can define the Mie-type potential as [13, 34]:

$$
V(r)=\kappa\left[\frac{a}{b-a}\left(\frac{r_{\mathrm{e}}}{r}\right)^{b}-\frac{b}{b-a}\left(\frac{r_{\mathrm{e}}}{r}\right)^{a}\right],
$$

where $a$ and $b$ are parameters, $\kappa$ is the interaction energy between two atoms in a molecular system at distance $r_{\mathrm{e}}$. An example on this type of potentials $(a=2, b=1)$ is the standard Morse [21] or Kratzer-Fues [22, 23] potential of the form $[15,20,24]$

$$
V(r)=-\kappa\left(\frac{2 r_{\mathrm{e}}}{r}-\frac{r_{\mathrm{e}}^{2}}{r^{2}}\right) .
$$

Moreover, the standard Kratzer potential is modified by adding a term to the potential. A new type of this potential is the modified Kratzer-type potential [15, 23]:

$$
V(r)=-\kappa\left(\frac{r-r_{\mathrm{e}}}{r}\right)^{2} .
$$

However, in order to give a complete solution of these two potentials, we consider a general form

$$
V(r)=-\frac{A}{r}+\frac{B}{r^{2}}+C .
$$

The potential appears to be more flexible due to the addition of the parameter $C$. With $A=\kappa r_{\mathrm{e}}, B=\kappa r_{\mathrm{e}}^{2}$ and $C=\kappa$, we have the modified Kratzer potential and the Kratzer-Fues potential can be obtained by setting $A=2 \kappa r_{\mathrm{e}}, B=\kappa r_{\mathrm{e}}^{2}$ and $C=0$. For brevity, inserting the potential (6) into the radial part $R_{n_{r} \ell}(r)$ of $\Psi_{n \ell m}(r, \Omega)$, we obtain

$$
\begin{aligned}
& R_{n_{r} \ell}^{\prime \prime}(r)+\frac{N-1}{r} R_{n_{r} \ell}^{\prime}(r)-\frac{\ell(\ell+N-2)}{r^{2}} R_{n_{r} \ell}(r) \\
& +\frac{2 \mu}{\hbar^{2}}\left(E+\frac{A}{r}-\frac{B}{r^{2}}-C\right) R_{n_{r} \ell}(r)=0,
\end{aligned}
$$

where $E$ is the energy eigenvalue, $\ell$ is the orbital angular momentum quantum number satisfying

$$
\Lambda_{N}^{2}(\Omega) Y_{\ell}^{m}(\Omega)+\ell(\ell+N-2) Y_{\ell}^{m}(\Omega)=0,
$$

where $Y_{\ell}^{m}(\Omega)$ are the hyperspherical harmonics.
By making the substitution,

$$
R_{n_{r} \ell}(r)=r^{-\frac{N-1}{2}} U_{n_{r} \ell}(r) \text {, }
$$

Eq. (7) becomes

$$
\begin{aligned}
& U_{n_{r} \ell}^{\prime \prime}(r)+\left[\frac{2 \mu}{\hbar^{2}}\left(E+\frac{A}{r}-\frac{B}{r^{2}}-C\right)-\frac{\ell(\ell+N-2)}{r^{2}}\right. \\
& \left.-\frac{N-1 N-3}{4 r^{2}}\right] U_{n_{r} \ell}(r)=0 .
\end{aligned}
$$

We note that Eq. (22) is similar to the one-dimensional Schrödinger except for the addition of the centrifugal term $\frac{\ell(\ell+N-2)}{r^{2}}$ to the potential. Defining the following parameters:

$$
\begin{aligned}
& t=\sqrt{\frac{8 \mu(C-E)}{\hbar^{2}}} r, \quad \alpha=\sqrt{\frac{\mu}{2 \hbar(C-E)}} A, \\
& \nu(\nu+N-2)=\ell(\ell+N-2)+\frac{2 \mu B}{\hbar^{2}},
\end{aligned}
$$

Eq. (7) becomes

$$
\begin{aligned}
& R_{n_{r} \ell}^{\prime \prime}(t)+\frac{N-1}{t} R_{n_{r} \ell}^{\prime}(t) \\
& \quad+\left[-\frac{1}{4}+\frac{\alpha}{t}-\frac{\nu(\nu+N-2)}{t^{2}}\right] R_{n_{r} \ell}(t)=0 .
\end{aligned}
$$

By the behaviour of the wave function at the origin and at infinity, we define

$$
R_{n_{r} \ell}(t)=\exp \left(-\frac{t}{2}\right) t^{\nu} f(t)
$$

The use of (13) transforms (12) to the well-known associated Laguerre differential equation

$$
\begin{array}{r}
t f^{\prime \prime}(t)+[(2 \nu+N-1)-t] f^{\prime}(t) \\
-\left(\nu+\frac{N-1}{2}-\alpha\right) f(t)=0 .
\end{array}
$$

Polynomial solution to (14) can be written in terms of the hypergeometric function as follows [31]:

$$
f(t)={ }_{1} F_{1}\left(\nu+\frac{N-1}{2}-\alpha, 2 \nu+N-1 ; t\right) .
$$

For large value of $t$, this solution diverges, thus preventing normalization. To prevent this, we set

$$
\nu+\frac{N-1}{2}-\alpha=-n_{r}, \quad n_{r}=0,1,2, \ldots
$$

where $n_{r}$ is the hyperradial quantum number. Thus, it is clear from (11) and (15b) that the energy eigenvalues can be obtained as

$$
\begin{aligned}
E_{n_{r}, \ell} & =C-\frac{2 \mu A^{2}}{\hbar^{2}\left(2 n_{r}+2 \nu+N-1\right)^{2}}, \\
n_{r}, \nu & =0,1,2, \ldots
\end{aligned}
$$

If we define the principal quantum number $n=n_{r}+\nu+1$, the energy Eq. (16) takes the following simple form: 


$$
E_{n}=C-\frac{2 \mu A^{2}}{\hbar^{2}(2 n+N+1)^{2}}, \quad n=1,2,3, \ldots
$$

The energy eigenvalues obtained in Eq. (16a) can be shown to be in agreement with those of the Kratzer-Fues potential by setting $C=0$, thus, we have

$$
E_{n_{r}, \ell}^{\mathrm{KF}}=-\frac{2 \mu A^{2}}{\hbar^{2}\left(2 n_{r}+2 \nu+N-1\right)^{2}} .
$$

Also, if $B$ and $C$ are set to be zero, Eq. (16a) becomes

$$
E_{n_{r}, \ell}^{\mathrm{Col}}=-\frac{2 \mu A^{2}}{\hbar^{2}\left(2 n_{r}+2 \ell+N-1\right)^{2}},
$$

which is the energy values for the Coulombic-type potential [39]. Numerical values of the energy for some diatomic molecules using the modified Kratzer-type potential (5) are given in Table I using the following potential parameters for $\mathrm{H}_{2}\left(\kappa=4.7446 \mathrm{eV}, r_{\mathrm{e}}=0.7416 \AA\right.$, $\mu=0.50391 \mathrm{amu}), \mathrm{HCl}\left(\kappa=4.6243 \mathrm{eV}, r_{\mathrm{e}}=1.2746 \AA\right.$, $\mu=0.9801045 \mathrm{amu})$ and $\mathrm{LiH}\left(\kappa=2.5181 \mathrm{eV}, r_{\mathrm{e}}=\right.$ $1.5956 \AA, \mu=0.8801221 \mathrm{amu})$.

TABLE I

The bound-state energy levels $E_{n}$ for some diatomic molecules using the modified Kratzer-type potential (5) for some dimensions and $\hbar=1$.

\begin{tabular}{c|c|c|c|c}
\hline \hline \multicolumn{5}{c}{$E_{n}[\mathrm{eV}]$} \\
\hline$N$ & $n$ & $\mathrm{H}_{2}$ & $\mathrm{HCl}$ & $\mathrm{LiH}$ \\
\hline \multirow{4}{*}{3} & 1 & -6.1310 & -12.1908 & -5.6755 \\
& 2 & -5.5244 & -8.8804 & -4.2942 \\
& 3 & -5.2437 & -7.3482 & -3.6548 \\
& 4 & -5.0912 & -6.5159 & -3.3075 \\
& 5 & -4.9992 & -6.0140 & -3.0981 \\
\hline \multirow{4}{*}{4} & 1 & -5.7632 & -10.1833 & -4.8379 \\
& 2 & -5.3608 & -7.9872 & -3.9214 \\
& 3 & -5.1571 & -6.8755 & -3.4575 \\
& 4 & -5.0399 & -6.2361 & -3.1907 \\
& 5 & -4.9664 & -5.8349 & -3.0233 \\
\hline \multirow{5}{*}{5} & 1 & -5.5244 & -8.8804 & -4.2942 \\
& 2 & -5.2437 & -7.3482 & -3.6548 \\
& 3 & -5.0912 & -6.5159 & -3.3075 \\
& 4 & -4.9992 & -6.0140 & -3.0981 \\
& 5 & -4.9396 & -5.6883 & -2.9621
\end{tabular}

Finally, using the following relation:

$$
{ }_{1} F_{1}(-a, b+1 ; x)=\frac{a ! b !}{(a+b) !} L_{a}^{b}(x),
$$

with Eqs. (11), (13) and (15), the wave function of the $N$-dimensional Schrödinger equation for the Mie-type potential is given as

$$
R_{n_{r} \ell}(r)=C_{n_{r}} r^{v-\frac{N-3}{2}} \mathrm{e}^{-\epsilon r} L_{n_{r}}^{2 v+1}(2 \epsilon r),
$$

where we have defined

$$
v=\frac{1}{2}\left[\sqrt{(2 \ell+N-2)^{2}+\frac{8 \mu B}{\hbar^{2}}}-1\right]
$$

and

$$
\epsilon=\frac{4 \mu A}{\hbar^{2}\left(2 n_{r}+1+\sqrt{(2 \ell+N-2)^{2}+\frac{8 \mu B}{\hbar^{2}}}\right)},
$$

and $C_{n_{r}}$ is the normalization constant to be determined.

Using the normalization condition

$$
\int_{0}^{\infty}\left|R_{n_{r} \ell}(r)\right|^{2} r^{N-1} \mathrm{~d} r=1
$$

and the orthogonal property of the Laguerre polynomials

$$
\int_{0}^{\infty} z^{\eta+1}\left[L_{n}^{\eta}(z)\right] \mathrm{d} z=\frac{(2 n+\eta+1)(n+\eta)}{n !},
$$

we obtain

$$
C_{n_{r}}=\sqrt{\frac{n_{r} !(2 \epsilon)^{2 v+3}}{2\left(n_{r}+v+1\right) n_{r}+2 v+1 !}} .
$$

We can now express the normalized wave function as

$$
\begin{aligned}
& R_{n_{r} \ell}(r)=\sqrt{\frac{n_{r} !(2 \epsilon)^{2 v+3}}{2\left(n_{r}+v+1\right) n_{r}+2 v+1 !}} \\
& \quad \times r^{v-\frac{N-3}{2}} \mathrm{e}^{-\epsilon r} L_{n_{r}}^{2 v+1}(2 \epsilon r) .
\end{aligned}
$$

Therefore the complete orthonormalized energy eigenfunctions of the Mie-type potential is given as

$$
\Psi_{n \ell m}(r, \theta, \varphi)=R_{n_{r} \ell}(r) Y_{\ell}^{m}(\Omega),
$$

where $R_{n_{r} \ell}(r)$ is given in Eq. (26) and $Y_{\ell}^{m}(\Omega)$ are the hyperspherical harmonics. Moreover, we note here that the wave function (26) reduces to those of the Coulombic-type when $B=0, C=0[39]$ and Kratzer-Fues when $C=0[2]$.

\subsection{Degeneracy of energy levels}

In this section, we give a simple presentation for determining the degeneracy of the energy levels of the Mie-type potentials in $N$-dimensions. If the potential under consideration (Eq. (6)) has no other symmetries beyond rotational invariance, the degeneracy of energy levels are therefore the multiplicities of the hyperspherical harmonics for a fixed $\nu$ (the new angular momentum defined in Eq. 11). In order to get a clear understanding of what the degenerate levels of the Mie-type potentials look like, we write the energy Eq. (16) as follows:

$$
E_{n}=C-\frac{\mu A^{2}}{2 \hbar^{2}\left(n+\frac{N-3}{2}\right)^{2}},
$$

where $n=n_{r}+\nu+1$. Equation (28) is analogous to that of the Coulombic energy levels except for the addition of the parameter $C$.

In $N$ dimensions, the hyperspherical harmonics depend on $N-1$ angular coordinates $\theta_{1}, \theta_{2}, \ldots \theta_{N-2}, \phi$ whose ranges are $0 \leq \theta_{j} \leq \pi$ and $0 \leq \phi \leq 2 \pi$. Also, each hyperspherical harmonic is determined by $N-1$ integers 
$\nu, m_{1}, m_{2}, \ldots m_{N-2}$. Thus by enumerating all the distinct set of $m$ value that are possible for a given $\nu$, the degeneracy is given by [39]:

$$
\text { degeneracy }=\sum_{\nu=0}^{n-1} \sum_{-\nu}^{\nu}\left|m_{1}\right|=\sum_{\nu=0}^{n-1}(2 \nu+1)=n^{2}
$$

for $N=3$, and

$$
\text { degeneracy }=\sum_{\nu=0}^{n-1} \sum_{m_{1}=0}^{\nu} \sum_{m_{2}=0}^{m_{1}} \ldots \sum_{-m_{N-3}}^{m_{N-3}}\left|m_{N-2}\right|
$$

for $N>3$. With the help of Eqs. (29) and (30), results of the degeneracies of the Mie-type potentials in $N$-dimensions are given in Table II for $N=3-10$ and $n=1-5$.

TABLE II

Degeneracies of the Mie-type potentials in $N$ dimensions for $N=3-10$ and $n=1-5$.

\begin{tabular}{c|c|c|c|c|c}
\hline \hline$N$ & $n=1$ & $n=2$ & $n=3$ & $n=4$ & $n=5$ \\
\hline 3 & 1 & 4 & 9 & 16 & 25 \\
4 & 1 & 5 & 14 & 30 & 55 \\
5 & 1 & 6 & 20 & 50 & 105 \\
6 & 1 & 7 & 27 & 77 & 182 \\
7 & 1 & 8 & 35 & 112 & 294 \\
8 & 1 & 9 & 44 & 156 & 450 \\
9 & 1 & 10 & 54 & 210 & 665 \\
10 & 1 & 11 & 65 & 275 & 935
\end{tabular}

\section{Some expectation values and the virial theorem for the Mie-type potential in $N$-dimensions}

In this section, we obtain the expectation values $\left\langle r^{-1}\right\rangle$ and $\left\langle r^{-2}\right\rangle$ directly using the Hellmann-Feynman theorem (HFT) [25-27]. Although the HFT is commonly used in the calculation of intermolecular forces in molecules, however, in order to employ the HFT in calculating the expectation values, one can promote the fixed parameters which appears in the Hamiltonian to be a continuous variable (for the mathematical purpose of taking the derivative). Thus, suppose the Hamiltonian $H$ for a particular quantum system is a function of some parameters $q$, and let $E_{n}(q)$ and $\Psi_{n}(q)$ be the eigenvalues and eigenfunctions of $H(q)$ respectively, then the HFT states that

$$
\frac{\partial E_{n}(q)}{\partial q}=\left\langle\Psi_{n}(q)\left|\frac{\partial H(q)}{\partial q}\right| \Psi_{n}(q)\right\rangle .
$$

The effective Hamiltonian of the hyperradial wave function is given as

$$
H=\frac{-\hbar^{2}}{2 \mu} \frac{\mathrm{d}^{2}}{\mathrm{~d} r^{2}}+\frac{-\hbar^{2}}{2 \mu} \frac{(2 \ell+N-1)(2 \ell+N-3)}{4 r^{2}}
$$

$$
-\frac{A}{r}+\frac{B}{r^{2}}+C
$$

To obtain $\left\langle r^{-1}\right\rangle$, we let $q=A$ such that

$$
\left\langle\Psi_{n}(A)\left|\frac{\partial H(A)}{\partial A}\right| \Psi_{n}(A)\right\rangle=\left\langle r^{-1}\right\rangle
$$

and

$$
\frac{\partial E_{n}(A)}{\partial A}=\frac{4 \mu A}{\hbar^{2}\left[2 n_{r}+1+\sqrt{(2 \ell+N-2)^{2}+\frac{8 \mu B}{\hbar^{2}}}\right]^{2}} .
$$

Thus by the HFT, we have

$$
\left\langle r^{-1}\right\rangle=\frac{4 \mu A}{\hbar^{2}\left[2 n_{r}+1+\sqrt{(2 \ell+N-2)^{2}+\frac{8 \mu B}{\hbar^{2}}}\right]^{2}} .
$$

Similarly, by letting $q=B$ in Eq. (28) we obtain $\left\langle r^{-2}\right\rangle$ as

$$
\begin{gathered}
\left\langle r^{-2}\right\rangle=\left(16 \mu^{2} A^{2}\right) /\left\{\hbar^{4} \sqrt{(2 \ell+N-2)^{2}+\frac{8 \mu B}{\hbar^{2}}}\right. \\
\left.\times\left[2 n_{r}+1+\sqrt{(2 \ell+N-2)^{2}+\frac{8 \mu B}{\hbar^{2}}}\right]^{3}\right\} .
\end{gathered}
$$

We note that $q=\ell$ also yields the same result for $\left\langle r^{-2}\right\rangle$.

Finally, if we let $q=\mu$, then by the HFT, we have the virial theorem of the Mie-type potential as follows:

$$
-\langle H-V\rangle=(1-\beta) E_{n} .
$$

Since $H=T+V=E_{n}$, we get

$$
-(2-\beta)\langle T\rangle=(1-\beta)\langle V\rangle,
$$

where

$$
\begin{aligned}
\beta & =(8 \mu B) /\left\{\hbar^{2} \sqrt{(2 \ell+N-2)^{2}+\frac{8 \mu B}{\hbar^{2}}}\right. \\
& \left.\times\left[2 n_{r}+1+\sqrt{(2 \ell+N-2)^{2}+\frac{8 \mu B}{\hbar^{2}}}\right]\right\} .
\end{aligned}
$$

\section{Ladder operators for Mie-type potential in $\boldsymbol{N}$-dimensions}

We shall now construct the creation and annihilation operator for the eigenfunctions obtained in (26) using the factorization method [28-30, 36, 37]. As shown in previous works, the ladder operators can be constructed directly from the wave function without introducing any auxiliary variable. Therefore, we intend to find differential operators $\hat{L}_{ \pm}$satisfying the property

$$
\hat{L}_{ \pm} R_{n_{r} \ell}(r)=\ell_{ \pm} R_{n_{r} \pm 1, \ell}(r) .
$$

In other words, we wish to find the operators of the form

$$
\hat{L}_{ \pm}=f_{ \pm}(r) \frac{\mathrm{d}}{\mathrm{d} r}+g_{ \pm}(r),
$$

which depend on the physical variable $r$. 
To this end, we start by obtaining the derivative of the wave function (26):

$$
\begin{aligned}
& \frac{\mathrm{d}}{\mathrm{d} r} R_{n_{r} \ell}(r)=\frac{R_{n_{r} \ell}(r)}{r}\left(v-\frac{N-3}{2}\right)-\epsilon R_{n_{r} \ell}(r) \\
& \quad+N_{n_{r}}^{v} r^{v-\frac{N-3}{2}} \mathrm{e}^{-\epsilon r} \frac{\mathrm{d}}{\mathrm{d} r} L_{n_{r}}^{2 v+1}(2 \epsilon r),
\end{aligned}
$$

where

$$
N_{n_{r}}^{v}=\sqrt{\frac{n_{r} !(2 \epsilon)^{2 v+3}}{2\left(n_{r}+v+1\right) n_{r}+2 v+1 !}} .
$$

With the use of the following relation of the associated Laguerre function [31]:

$$
x \frac{\mathrm{d}}{\mathrm{d} x} L_{n}^{\alpha}(x)=n L_{n}^{\alpha}(x)-(n+\alpha) L_{n-1}^{\alpha},
$$

Eq. (43) becomes

$$
\begin{gathered}
{\left[-\frac{\mathrm{d}}{\mathrm{d} r}-\epsilon+\frac{1}{r}\left(n_{r}+v-\frac{N-3}{2}\right)\right] R_{n \ell}(r)} \\
=\frac{n_{r}+2 v+1}{r} \frac{N_{n_{r}}^{v}}{N_{n_{r}-1}^{v}} R_{n-1, \ell}(r) .
\end{gathered}
$$

Using (43), we have

$$
\begin{gathered}
{\left[-r \frac{\mathrm{d}}{\mathrm{d} r}-\epsilon r+\left(n_{r}+v-\frac{N-3}{2}\right)\right] R_{n_{r} \ell}(r)} \\
=\sqrt{\frac{n_{r}\left(n_{r}+v\right) n_{r}+2 v+1}{n_{r}+v+1}} R_{n_{r}-1, \ell}(r) .
\end{gathered}
$$

Hence the annihilation operator is defined as

$$
\hat{L}_{-}=\left[-r \frac{\mathrm{d}}{\mathrm{d} r}-\epsilon r+\left(n_{r}+v-\frac{N-3}{2}\right)\right]
$$

with the following effect on the wave function

$$
\hat{L}_{-} R_{n_{r} \ell}(r)=\ell_{-} R_{n_{r}-1, \ell}(r),
$$

where

$$
\ell_{-}=\sqrt{\frac{n_{r}\left(n_{r}+v\right) n_{r}+2 v+1}{n_{r}+v+1}} .
$$

Similarly, the creation operator can be obtained by using the following property of the associated Laguerre polynomial [31] in Eq. (42):

$$
x \frac{\mathrm{d}}{\mathrm{d} x} L_{n}^{\alpha}(x)=(n+1) L_{n+1}^{\alpha}(x)-(n+\alpha+1-x) L_{n}^{\alpha} .
$$

Thus, we have

$$
\begin{aligned}
& {\left[\frac{\mathrm{d}}{\mathrm{d} r}+\epsilon+\frac{1}{r}\left(n_{r}+v-2 \epsilon r-\frac{N-1}{2}\right)\right] R_{n_{r} \ell}(r)} \\
& \quad=\frac{n_{r}+1}{r} \frac{N_{n_{r}}^{v}}{N_{n_{r}+1}^{v}} R_{n_{r}+1, \ell}(r) .
\end{aligned}
$$

Also, using (43), we have

$$
\left[r \frac{\mathrm{d}}{\mathrm{d} r}-\epsilon r+\left(n_{r}+v-\frac{N-1}{2}\right)\right] R_{n_{r} \ell}(r)
$$

$$
\begin{aligned}
& =\sqrt{\frac{n_{r}+1\left(n_{r}+v+2\right)\left(n_{r}+2 v+2\right)}{n_{r}+v+1}} \\
& \times R_{n_{r}+1, \ell}(r),
\end{aligned}
$$

whence, we can define the creator operator as

$$
\hat{L}_{+}=\left[r \frac{\mathrm{d}}{\mathrm{d} r}-\epsilon r+\left(n_{r}+v-\frac{N-1}{2}\right)\right]
$$

satisfying

$$
\hat{L}_{+} R_{n_{r} \ell}(r)=\ell_{+} R_{n_{r}+1, \ell}(r)
$$

with

$$
\ell_{+}=\sqrt{\frac{n_{r}+1\left(n_{r}+v+2\right)\left(n_{r}+2 v+2\right)}{n_{r}+v+1}} .
$$

At this point, it is important to stress that operators (47) and (53) as they have been derived above are not constructed as the factorizing operators of the Hamiltonian. It is well known that the factorization operators are not, in general, the ladder operator of the system (for details see Refs. [35-38]).

We now turn our attention to the algebra associated with the operators $\hat{L}_{-}$and $\hat{L}_{+}$. From Eqs. (47) and (53), we can compute the commutator $\left[\hat{L}_{-}, \hat{L}_{+}\right]$as follows:

$$
\begin{aligned}
& {\left[\hat{L}_{-}, \hat{L}_{+}\right] R_{n_{r} \ell}(r)} \\
& =\hat{L}_{-}\left\{\hat{L}_{+} R_{n_{r} \ell}(r)\right\}-\hat{L}_{+}\left\{\hat{L}_{-} R_{n_{r} \ell}(r)\right\} \\
& \quad=\sqrt{\frac{n_{r}+1\left(n_{r}+v+2\right)\left(n_{r}+2 v+2\right)}{n_{r}+v+1}} \\
& \quad \times\left\{\hat{L}_{-}, R_{n_{r}+1, \ell}(r)\right\} \\
& \quad-\sqrt{\frac{n_{r}\left(n_{r}+v\right) n_{r}+2 v+1}{n_{r}+v+1}} \\
& \quad \times\left\{\hat{L}_{+}, R_{n_{r}-1, \ell}(r)\right\} \\
& =\left(2 n_{r}+2 v+2\right) R_{n_{r} \ell}(r)=2 \ell_{0} R_{n_{r} \ell}(r),
\end{aligned}
$$

where we have introduced the eigenvalue

$$
\ell_{0}=n_{r}+v+1 \text {. }
$$

We can thus define the operator

$$
\hat{L}_{0}=\hat{n}_{r}+v+1 \text {. }
$$

It is easy to show that the operators $\hat{L}_{ \pm}$and $\hat{L}_{0}$ satisfy the commutator relations

$$
\begin{aligned}
& {\left[\hat{L}_{-}, \hat{L}_{+}\right]=2 \hat{L}_{0}, \quad\left[\hat{L}_{0}, \hat{L}_{-}\right]=-\hat{L}_{-},} \\
& {\left[\hat{L}_{0}, \hat{L}_{+}\right]=\hat{L}_{+},}
\end{aligned}
$$

which correspond to the commutator relations of the dynamic group $\mathrm{SU}(1,1)$. Using Eq. (58), the Hamiltonian now acquires a simple form 


$$
H=C-\frac{\mu A^{2} / 2 \hbar^{2}}{\hat{L}_{0}^{2}} .
$$

Moreover, the following expressions can easily be obtained for the operators:

$$
\begin{aligned}
& r R_{n_{r} \ell}(r)=\frac{1}{2 \epsilon}\left[2 \hat{L}_{0}-\left(\hat{L}_{+}+\hat{L}_{-}\right)\right] R_{n_{r} \ell}(r) \\
& -\frac{N}{2 \epsilon} R_{n_{r} \ell}(r)
\end{aligned}
$$

and

$$
r \frac{\mathrm{d}}{\mathrm{d} r} R_{n_{r} \ell}(r)=\frac{1}{2}\left(\hat{L}_{+}-\hat{L}_{-}\right) R_{n_{r} \ell}(r)-\frac{1}{2} R_{n_{r} \ell}(r) .
$$

Also, the corresponding matrix elements of these two functions can be obtained as

$$
\begin{gathered}
\left\langle R_{n_{r} \ell}(r)|r| R_{m_{r} \ell}(r)\right\rangle=\frac{1}{2 \epsilon}\left[\left(2 n_{r}+2 v+2\right) \delta_{m_{r}, n_{r}}\right. \\
\left.-\ell_{+} \delta_{m_{r}, n_{r}+1}-\ell_{-} \delta_{m_{r}, n_{r}-1}\right]-\frac{N}{2 \epsilon} \delta_{m, n}
\end{gathered}
$$

and

$$
\begin{aligned}
& \left\langle R_{n_{r} \ell}(r)\left|r \frac{\mathrm{d}}{\mathrm{d} r}\right| R_{m_{r} \ell}(r)\right\rangle=\frac{1}{2}\left[\ell_{+} \delta_{m_{r}, n_{r}+1}\right. \\
& \left.-\ell_{-} \delta_{m_{r}, n_{r}-1}\right]-\frac{1}{2} \delta_{m_{r}, n_{r}},
\end{aligned}
$$

respectively.

Finally, the Casimir operator [32] can also be expressed as

$$
\begin{aligned}
\tilde{C} & R_{n_{r} \ell}(r)=\left[\hat{L}_{0}\left(\hat{L}_{0}-1\right)-\hat{L}_{+} \hat{L}_{-}\right] R_{n_{r} \ell}(r) \\
\quad= & {\left[\hat{L}_{0}\left(\hat{L}_{0}+1\right)-\hat{L}_{-} \hat{L}_{+}\right] R_{n_{r} \ell}(r) } \\
& =J(J-1) R_{n_{r} \ell}(r),
\end{aligned}
$$

where

$$
J=v+1 .
$$

\section{Conclusions}

In this paper, the solution to the $N$-dimensional Schrödinger equation with the Mie-type potentials was obtained. The energy eigenvalues obtained were found to be consistent with those of the 3-dimensional Mie-type potential when $N=3$, also the energy levels reduces to those of the Coulombic-type when $B=0$. The energy equation obtained was also used to obtain the vibrational energy values of some diatomic molecules bounded by a modified Kratzer-type potential. The eigenfunctions were also worked out in terms of the associated Laguerre polynomials and were shown to be in agreement with those obtained in literature as $N=3$.

Moreover, expectation values $\left\langle r^{-1}\right\rangle$ and $\left\langle r^{-2}\right\rangle$ were calculated using the Hellmann-Feynman theorem and the values obtained indicate the clustered of more electrons around the Coulombic part of the potential. The virial theorem was also verified for the Mie-type potentials in $N$-dimensions and the result obtained reduces to that of the usual virial theorem $-2\langle T\rangle=\langle V\rangle$ when $B=0$, as expected for a Coulombic potential.

Also, using the factorization method, the creation and annihilation operators were also constructed for the Mie- -type potential and they were found to agree with those of the 3-dimensional Coulombic potential when $N=3$ and $B=0[29]$; moreover, the operators were shown to obey the $\mathrm{SU}(1,1)$ algebra. However, we stress that the operators (47) and (53) as constructed in the work are not the factorizing operators of the Hamiltonian.

We also observed that the degeneracy of the energy levels increases with the dimension $N$. Finally, that note that with a proper choice of the parameters, the results obtained become those of the modified Kratzer and the Kratzer-Fues potentials.

\section{Acknowledgments}

D.A. wishes to thank Profs. R. Sever, L.M. Nieto, and O. Rosas-Ortiz for communicating to him some of their works during the preparation of the manuscript. He also wishes to thank the referee for pointing his attention to some useful references and he is also indebted to Father J. and Agboola B. for their encouragements.

\section{References}

[1] D.S. Bateman, C. Boyd, B. Dutta-Roy, Am. J. Phys. 60, 833 (1992).

[2] H. Hassanabadi, S. Zarrinkamar, A.A. Rajabi, Commun. Theor. Phys. 55, 541 (2011).

[3] K.J. Oyewumi, F.O. Akinpelu, A.D. Agboola, Int. J. Theor. Phys. 47, 1039 (2008).

[4] D. Agboola, Phys. Scr. 80, 065304 (2009).

[5] D. Agboola, Phys. Scr. 81, 067001 (2010).

[6] D. Agboola, Chin. Phys. Lett. 27, 040301 (2010).

[7] Y.C. Chen, S.H. Dong, Phys. Lett. A 335, 374 (2005).

[8] F.T. Cheng, T.Q. Dai, Phys. Scr. 75, 274 (2007).

[9] S. Ikhdair, R. Sever, Int. J. Mod. Phys. C 19, 221 (2008).

[10] A.F. Nikiforov, V.B. Uvarov, Special Functions of Mathematical Physics, Birkhauser, Bassel 1988.

[11] S. Ikhdair, R. Sever, Chin. J. Phys. 46, 291 (2008).

[12] S. Ikhdair, R. Sever, Cent. Eur. J. Phys. 6, 685 (2008).

[13] S. Ikhdair, R. Sever, J. Math. Chem. 42, 461 (2007).

[14] S. Ikhdair, R. Sever, Int. J. Mod. Phys. C 20, 361 (2009).

[15] S. Ikhdair, R. Sever, Cent. Eur. J. Phys. 6, 697 (2008).

[16] M. Hamzavi, A.A. Rajabi, Commun. Theor. Phys. 55, 35 (2011).

[17] S. Ikhdair, R. Sever, Int. J. Mod. Phys. C 19, 1425 (2008).

[18] J. Avery, Hyperspherical Harmonics: Application in Quantum Theory, Kluwer, Dordrecht 1989.

[19] E. Schrödinger, Proc. R. Irish Acad. A 46, 183 (1940).

[20] S.M. Ikhdair, R. Sever, arXiv: quant-ph/0611065.

[21] P.M. Morse, Phys. Rev. 34, 57 (1929).

[22] A. Kratzer, Z. Phys. 3, 289 (1920). 
[23] E. Fues, Ann. Phys. 80, 367 (1926).

[24] C. Berkdemir, A. Berkdemir, J. Han, Chem. Phys. Lett. 417, 326 (2006).

[25] G. Hellmann, Einführung in die Quantenchemie, Denticke, Vienna 1937 (in German).

[26] R.P. Feynman, Phys. Rev. 56, 340 (1939).

[27] W.G. McMillan, G. Marc, Adv. Chem. Phys. 58, 205 (1985).

[28] S.H. Dong, Comp. Math. Appl. 47, 1035 (2004).

[29] L.I. Cooper, J. Phys. A, Math. Gen. 25, 1671 (1992).

[30] L.I. Cooper, J. Phys. A, Math. Gen. 26, 1601 (1993).

[31] I.S. Gradshteyn, I.M. Ryzhik, Tables of Integrals, Series, and Products, 5th ed. Academic Press, New York 1994.
[32] H. Casimir, Proc. R. Acad. 34, 844 (1931).

[33] S. Erkoc, R. Sever, Phys. Rev. D 33, 588 (1986).

[34] S. Erkoc, R. Sever, Phys. Rev. D 30, 2117 (1984).

[35] F.M. Fernandez, S.A. Maluendes, E.A. Castro, Phys. Rev. D 36, 650 (1987).

[36] B. Mielnik, O. Rosas-Ortiz, J. Phys. A, Maths. Gen. 37, 10007 (2004).

[37] J. Negro, L.M. Nieto, O. Rosas-Ortiz, J. Phys. A, Maths. Gen. 33, 7207 (2000).

[38] J. Negro, L.M. Nieto, O. Rosas-Ortiz, J. Math. Phys. 41, 7964 (2000).

[39] S.M. Al-Jaber, Int. J. Theor. Phys. 37, 1289 (1998). 